\title{
Perinatal exposure to bisphenol A exacerbates nonalcoholic steatohepatitis-like phenotype in male rat offspring fed on a high-fat diet
}

\author{
Jie Wei ${ }^{2, *}$, Xia Sun ${ }^{1, *}$, Yajie Chen', Yuanyuan Li, Liqiong Song, Zhao Zhou, Bing Xu, \\ Yi Lin ${ }^{1}$ and Shunqing $X u$
}

Correspondence should be addressed to $\mathrm{S} X \mathrm{Xu}$ or $\mathrm{Y}$ Lin Emails xust@hust.edu.cn or ylin@iue.ac.cn

\begin{abstract}
Bisphenol A (BPA) is one of the environmental endocrine disrupting chemicals, which is present ubiquitously in daily life. Accumulating evidence indicates that exposure to BPA contributes to metabolic syndrome. In this study, we examined whether perinatal exposure to BPA predisposed offspring to fatty liver disease: the hepatic manifestation of metabolic syndrome. Wistar rats were exposed to $50 \mu \mathrm{g} / \mathrm{kg}$ per day BPA or corn oil throughout gestation and lactation by oral gavage. Offspring were fed a standard chow diet (SD) or a high-fat diet (HFD) after weaning. Effects of BPA were assessed by examination of hepatic morphology, biochemical analysis, and the hepatic expression of genes and/or proteins involved in lipogenesis, fatty acid oxidation, gluconeogenesis, insulin signaling, inflammation, and fibrosis. On a SD, the offspring of rats exposed to BPA exhibited moderate hepatic steatosis and altered expression of insulin signaling elements in the liver, but with normal liver function. On a HFD, the offspring of rats exposed to BPA showed a nonalcoholic steatohepatitis-like phenotype, characterized by extensive accumulation of lipids, large lipid droplets, profound ballooning degeneration, impaired liver function, increased inflammation, and even mild fibrosis in the liver. Perinatal exposure to BPA worsened the hepatic damage caused by the HFD in the rat offspring. The additive effects of BPA correlated with higher levels of hepatic oxidative stress. Collectively, exposure to BPA may be a new risk factor for the development of fatty liver disease and further studies should assess whether this finding is also relevant to the human population.
\end{abstract}

\footnotetext{
Key Words

- bisphenol A

- steatosis

- insulin resistance

- oxidative stress

- inflammation

- fibrosis
}

Journal of Endocrinology (2014) 222, 313-325
() 2014 Society for Endocrinology Printed in Great Britain
Published by Bioscientifica Ltd 


\section{Introduction}

Nonalcoholic fatty liver disease (NAFLD) is one of the typical hepatic manifestations of metabolic syndrome characterized by hepatic fat accumulation in the absence of excess alcohol consumption. NAFLD affects $15-40 \%$ of the general population in Western countries and 9-40\% of people in Asian countries (Farrell \& Larter 2006). A subset of patients with NAFLD may progress to nonalcoholic steatohepatitis (NASH), a more severe form of hepatic damage associated with inflammation, fibrosis, cirrhosis, or hepatocellular carcinoma (Farrell \& Larter 2006). In addition to genetic and diet-related factors, exposure to environmental pollutants has been shown to negatively affect key pathogenic events implicated in NAFLD. For instance, chronic inhalation of low levels of ambient air particulate matter (PM 2.5) has been was reported to induce hepatic steatosis and lipid peroxidation and, moreover, increase hepatic inflammatory and fibrosis stage in mice (Tomaru et al. 2007, Tan et al. 2009). Exposure to nitrosamines and nicotine has also been found to play critical roles in the pathogenesis of NAFLD (Tong et al. 2009, Azzalini et al. 2010, Friedman et al. 2012).

Bisphenol A (BPA) is a well-known environmental endocrine disrupting chemical, which is used extensively in the manufacture of products containing epoxy resins and polycarbonate, such as toys, food and beverage containers, paper currency, etc. (Diamanti-Kandarakis et al. 2009). Humans can be exposed to BPA present in commonly used products. Recently, growing evidence has indicated that exposure to BPA is associated with an increased risk of some metabolic diseases such as obesity, insulin resistance, type 2 diabetes, dyslipidemia, etc. (Alonso-Magdalena et al. 2011, Shankar et al. 2012, Vom Saal et al. 2012). The presence of these metabolic disorder events has been documented to predict the future development of NAFLD (Fan et al. 2005, Hamaguchi et al. 2005). Indeed, a recent epidemiological study has related BPA exposure to abnormal concentrations of the liver enzymes $\gamma$-glutamyltransferase $(\gamma \mathrm{GT})$ and alkaline phosphatase (ALP), two of the sensitive markers for hepatocellular damage (Lang et al. 2008). In addition, low concentrations of BPA $\left(10^{-4}-10^{-12} \mathrm{M}\right)$ have been reported to induce lipid accumulation and steatosis in hepatic HepG2 cells by disturbing mitochondrial function and releasing proinflammatory cytokines (Huc et al. 2012). An animal study also confirmed that oral exposure of adult male CD1 mice to BPA $(0,5,50,500$, and $5000 \mu \mathrm{g} / \mathrm{kg}$ per day) resulted in the accumulation of hepatic triglycerides (TGs) and cholesteryl esters (CHOL), changes in hepatic free fatty acid (FFA) composition as well as upregulation of genes involved in lipid biosynthesis (Marmugi et al. 2012). Most importantly, this study revealed a nonmonotonic dose-response curve in which lower doses of BPA (doses within one order of magnitude of approximately $50 \mu \mathrm{g} / \mathrm{kg}$ per day) were more effective than the higher dose $(5000 \mu \mathrm{g} / \mathrm{kg}$ per day) for altering lipid biosynthesis pathways.

Exposure to an adverse environment during critical windows of development will affect the programing of metabolically active tissues in offspring, predisposing individuals to metabolic disorders later in life. For example, maternal nutrient excess or restriction has been confirmed to increase the risk of NAFLD in the offspring (McCurdy et al. 2009, Bayol et al. 2010, Oben et al. 2010). For BPA, results from several studies using animal models indicated that metabolic disorders were observed in offspring when they were exposed to BPA during the critical period of development. Perinatal exposure to BPA altered early adipogenesis and increased the body weight of offspring (Rubin \& Soto 2009, Somm et al. 2009). A brief exposure to BPA during pregnancy has also been found to initiate insulin resistance in adult offspring (Alonso-Magdalena et al. 2010). Consistent with these findings, results from our previous study have confirmed that perinatal exposure to BPA resulted in a higher body weight and body fat percentage, a greater mass of white adipocytes, hyperlipidemia, hyperleptinemia, and insulin intolerance in adult rat offspring (Wei et al. 2011). All of these detrimental effects of BPA might increase the risk of NAFLD.

In this study, we employed a developmental BPA exposure paradigm in rats to test the hypothesis that perinatal exposure to BPA caused a predisposition to hepatic steatosis. Our findings supported this hypothesis and further indicated that perinatal exposure to BPA coupled with the postweaning high-fat diet (HFD) significantly initiated hepatic oxidative stress, thereby predisposing offspring to hepatic inflammation and early fibrosis in adulthood.

\section{Materials and methods}

\section{Ethics}

All animal experiments were carried out humanely following the guidelines for the care and use of animals established by Tongji Medical College and were approved by the Ethics Committee of Tongji Medical College.

Published by Bioscientifica Ltd. 


\section{Animals}

Wistar rats were housed in polypropylene cages with free access to food and water under standard conditions $\left(22 \pm 2{ }^{\circ} \mathrm{C}, 12 \mathrm{~h}\right.$ light:12 $\mathrm{h}$ darkness cycles). Glass water bottles were used to avoid potential contamination from sources other than administration. Pregnant female rats were administered corn oil or $50 \mu \mathrm{g} / \mathrm{kg}$ per day BPA by oral gavage from gestational day 0 to postnatal day 21. Offspring were culled to five males and five females in each litter after delivery and were nursed with their own mother. After weaning, offspring were supplied with a standard chow diet (SD) or a HFD and allowed to feed ad libitum. The SD contained $20.5 \%$ crude protein, $4.62 \%$ crude fat, and $52.5 \%$ nitrogen-free extract (total calories $3.45 \mathrm{kcal} / \mathrm{g}, 12.05 \%$ calories in fat). The HFD contained $23.15 \%$ crude protein, $13.14 \%$ crude fat, and 50.94\% nitrogen-free extract (total calories $4.15 \mathrm{kcal} / \mathrm{g}, 28.53 \%$ calories in fat). Both standard chow and HFDs were purchased from Shanghai Slac Laboratory Animal Co., Ltd. (Shanghai, China). All experiments were carried out on male offspring from different litters in each treatment group.

\section{Sample processing and morphology examination}

At the age of 27 weeks, rat offspring were killed and livers were quickly dissected out and weighed. Portions of the liver lobes were fixed in $4 \%$ paraformaldehyde, embedded in paraffin, and stained with hematoxylin/eosin (H\&E) for histological analyses. Liver sections were also stained by Masson's trichrome to visualize hepatic collagen deposition. The remaining liver samples were frozen in liquid nitrogen and stored at $-80^{\circ} \mathrm{C}$ for subsequent lipid assays and molecular analysis.

\section{Hepatic lipid and oxidative stress index assays}

Hepatic lipids were extracted according to Folch's methods (Folch et al. 1957). Briefly, $200 \mathrm{mg}$ of hepatic tissues were homogenized in PBS, and then were extracted with 2:1 (vol/vol) chloroform/methanol by shaking overnight at $4{ }^{\circ} \mathrm{C}$. The fractions of the organic phase were isolated, evaporated under nitrogen, resuspended in chloroform, and then were assayed for TGs, CHOL, FFAs, and phospholipids (PPLs) using commercially available kits (Mindray, Shenzhen, China) on a Hitachi 747 autoanalyzer. Oxidative stress markers including malondialdehyde (MDA), glutathione (GSH), oxidized glutathione (GSSG), and antioxidant enzymes (superoxide dismutase (SOD), glutathione peroxidase (GPx), catalase
(CAT) and glutathione S-transferase (GST)) in hepatic tissue homogenates were detected using the corresponding assay kits (Nanjing Jiancheng Bioengineering Institute, Nanjing, China).

\section{Liver function tests}

At the age of 27 weeks, blood was collected from the orbital sinus in rat offspring for serum measurements following $16 \mathrm{~h}$ of fasting. The liver biomarkers, namely alanine aminotransferase (ALT), aspartate aminotransferase (AST), ALP, and $\gamma \mathrm{GT}$, were assayed using commercially available kits (Mindray) on a Hitachi 747 autoanalyzer. In addition, the serum TNF and IL6 levels were measured using the rat Quantikine ELISA kit (R\&D Systems, Minneapolis, MN, USA) according to the manufacturer's instructions.

\section{Homeostasis Model Assessment of Insulin Resistance}

Homeostasis Model Assessment of Insulin Resistance (HOMA-IR) and quantitative insulin sensitivity check index (QUICKI) were calculated from fasting blood glucose and serum insulin values. HOMA-IR was calculated as fasting glucose $(\mathrm{mM} / \mathrm{l}) \times$ fasting insulin $(\mu \mathrm{U} / \mathrm{ml}) / 22.5$ (Matthews et al. 1985, Bonora et al. 2000). QUICKI was calculated as $1 /(\ln$ (fasting insulin, $(\mu \mathrm{U} / \mathrm{ml})+\ln$ (fasting glucose, mg/dl)) (Katz et al. 2000, Chen et al. 2005).

\section{RNA preparation and mRNA quantification}

Total RNA was extracted from frozen liver samples using TRIzol reagent (Invitrogen) and was reverse transcribed using a RevertAid First Strand cDNA Synthesis Kit (Thermo Scientific, Rockford, IL, USA) according to the manuals. cDNA was amplified by real-time quantitative PCR using the SYBR Green PCR Master Mix (Applied Biosystems) on an ABI Prism 7900 sequence detection system (Applied Biosystems). Relative mRNA expression levels were determined by the $2^{-\Delta \Delta C t}$ method with $\beta$-actin as an internal reference. The primer sequences are given in Table 1 .

\section{Protein extraction and western blot analysis}

Protein was extracted from liver using lysis buffer containing $50 \mathrm{mM}$ Tris- $\mathrm{HCl}(\mathrm{pH} 7.4), 150 \mathrm{mM} \mathrm{NaCl}$, 0.1\% SDS, $1 \mathrm{mM}$ EDTA, 1\% Triton X-100, $1 \mathrm{mM}$ phenylmethylsulfonyl fluoride, and cocktails of protease and phosphatase inhibitors. Protein concentration was determined using the BCA Protein Assay kit (Thermo Scientific, Rockford, IL, USA). For insulin signaling

Published by Bioscientifica Ltd. 
Table 1 Primer sequences for quantitative real-time PCR

\begin{tabular}{lc}
\hline Gene names & Size $(\mathrm{bp})$ \\
\cline { 1 - 2 } Acta2 & 146 \\
Actb & 153 \\
Cd36 & 128 \\
Col1a1 & 88 \\
Cpt1a & 101 \\
Fasn & 139 \\
G6pc & 99 \\
Cybb & 159 \\
Il6 & 133 \\
Cyba & 91 \\
Ncf1 & 149 \\
Pckl & 80 \\
Ppara & 122 \\
Pparg & 80 \\
Srebf1 & 97 \\
Tgfb1 & 97 \\
Tnf & 100 \\
&
\end{tabular}

\begin{tabular}{l}
\hline 5'-Primer \\
\hline CGAAGCGCAGAGCAAGAGAGGG \\
CAACGGCTCCGGCATGTGC \\
CTCTGACATTTGCAGGTCCA \\
CCTGCTGGTCCTAAGGGAGAGC \\
AAGGTGCTGCTCTCCTACCA \\
AGCCCAGGAACAACTCATCC \\
GGGCATCAATCTCCTCTGGG \\
CGTGGAGTGGTGTGTGAATGCC \\
CCCAACTTCCAATGCTCTCC \\
CAGATCGAGTGGGCCATGTGGG \\
CCCTGCATCCTATTGGAGCCC \\
CGGTGGGAACTCACTGCTTG \\
GGGATCTTAGAGGCGAGCCA \\
CTGACCCAATGGTTGCTGATTAC \\
CATCGACTACATCCGCTTCTTACA \\
GCTAATGGTGGACCGCAACA \\
CCATGGCCCAGACCCTCACA
\end{tabular}

3'-Primer
GCAAGGTCGGATGCTCCTCTGG
CTCTTGCTCTGGGCTCG
AGTGGTTGTCTGGGTTCTGG
CTCTCTCACCAGGCAGACCTCG
CGACCTGAGAGGACCTTGAC
ACACAGGGACCGAGTAATGC
TCCAGGACCCACCAATACGG
GCCAACCGAGTCACAGCCACAT
GGTTGCCGAGTAGACCTCA
AGCGTCCCGCAGTAGCCACGAT
CCTCGATGGCTTCACCCTCAGA
CCAGCCACCCTTCCTCCTTA
GGGCCACAGAGCACCAATCT
GGACGCAGGCTCTACTTTGATC
GTCTTTCAGTGATTTGCTTTTGTGA
TGCTTCCCGAATGTCTGACG
TCCAGCTGCTCCTCCGCTTG

Accession number

NM_031004.2

NM 031144.3

NM_031561.2

NM_053304.1

NM_031559.2

NM_017332.1

NM 013098.2

NM_023965.1

NM 012589.1

NM_024160.1

NM_053734.2

NM_198780.3

NM_013196.1

NM 001145366.1

XM_213329.5

NM 021578.2

NM_012675.3

Acta2, smooth muscle $\alpha$ actin; Actb, $\beta$ actin; Cd36, fatty acid translocase; Col1a1, collagen, type I, $\alpha 1$; Cpt1a, carnitine palmitoyltransferase $1 \alpha ;$ Fasn, fatty acid synthase; II6, interleukin 6; G6pc, glucose 6-phosphatase, catalytic subunit; Cybb, cytochrome b-245, $\beta$ polypeptide; Cyba, cytochrome b-245, $\alpha$ polypeptide; Ncf1, neutrophil cytosolic factor $1 ;$ Pckl, phosphoenolpyruvate carboxykinase 1 (soluble); Ppara, peroxisome proliferator-activated receptor $\alpha$; Pparg, peroxisome proliferator-activated receptor $\gamma ;$ Srebf1, sterol regulatory element-binding protein $1 ;$ Tgfb1, transforming growth factor, $\beta 1 ;$ Tnf, tumor necrosis factor $\alpha$.

experiments, rat offspring were fasted overnight, anesthetized with pentobarbital, and received intraperitoneal injections of $0.75 \mathrm{U} / \mathrm{kg}$ biosynthetic human insulin (Novolin R; Novo Nordisk AIS, Bagsvaerd, Denmark). After 5-10 min, the livers were harvested and the protein was prepared as described above.

For western blots, equal amounts of protein $(40 \mu \mathrm{g})$ were resolved with SDS-PAGE and electrophoretically transferred onto PVDF membranes (Bio-Rad). The membranes were blocked with $5 \%$ non-fat dehydrated milk and then were incubated with the primary antibody at $4{ }^{\circ} \mathrm{C}$ overnight. The following day, the membranes were incubated with the HRP-conjugated secondary antibody (Cell Signaling Technology, Beverly, MA, USA) at 1:2000 dilutions. For immunodetection, membranes were visualized using enzyme-catalyzed chemiluminescence (Amersham Biosciences). The density of the bands was quantified using the Image Pro Plus version 6.0 software (Media Cybernetics, Bethesda, MD, USA) and normalized to ACTB. The following primary antibodies were used: ACTB (Cell Signaling Technology, \#4970, 1:2000); AKT1 (Cell Signaling Technology, \#9272, 1:2000); Thr308phosphorylated AKT1 (Cell Signaling Technology, \#9275, 1:500); GSK3B (Cell Signaling Technology, \#9315, 1:2000); Ser9-phosphorylated GSK3B (Cell Signaling Technology, \#9336, 1:500); insulin receptor (INSR) (Cell Signaling Technology, \#3025, 1:1000); Tyr1150/1151-phosphorylated INSR (Cell Signaling Technology, \#2969, 1:500); IL6 (Abcam, Cambridge, MA, USA, ab8339, 1:1000); TNF
(Abcam, ab66579, 1:1000); Collagen1a1 (COL1A1) (Santa Cruz Biotechnology, Santa Cruz, CA, USA, sc-8784, 1:1000); SREBP1C (Santa Cruz Biotechnology, sc-8984, 1:100); G6PC (Santa Cruz, sc-25840, 1:500); PEPCK (Santa Cruz Biotechnology, sc-166778, 1:500); PPARA (Santa Cruz Biotechnology, sc-9000, 1:500); TGFB1 (Abcam, ab92468, 1:2000), and ACTA2 (Abcam, ab5694, 1:2000).

\section{Statistical analysis}

Data are expressed as mean \pm s.E.M. Statistical analysis was performed using the SPSS 17.0 software (SPSS, Inc.). Oneway ANOVA followed by Bonferroni's post hoc test was used for comparison of the relevant groups (Chan et al. 2013, Shen et al. 2014). A $P$ value of $<0.05$ was considered significant.

\section{Results}

\section{Body and liver weight and hepatic lipid content}

As shown in Fig. 1A, the body weight was significantly increased in offspring of rats exposed to BPA, regardless of whether they were fed on a standard chow or HFD. There was no difference in either liver weight or calculated liver/body weight ratio between control offspring and offspring of rats exposed to BPA fed on a SD (Fig. 1A). Compared with controls fed on a HFD, offspring of rats exposed to BPA fed on a HFD displayed 154 and 132\% increases in the liver weight and the calculated liver/body

Published by Bioscientifica Ltd 
A
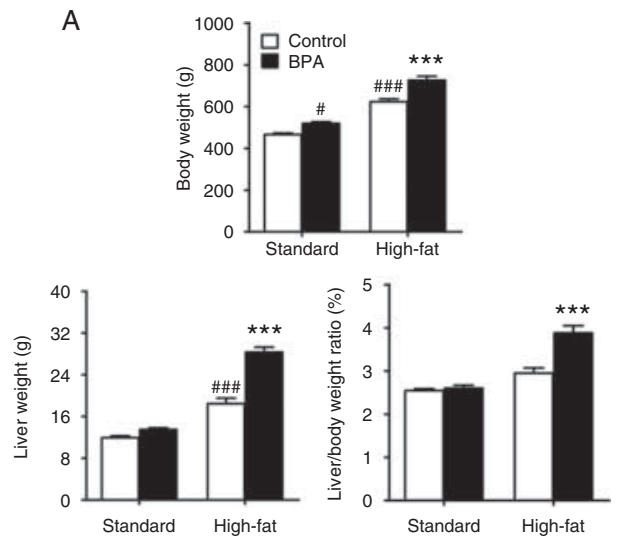

C
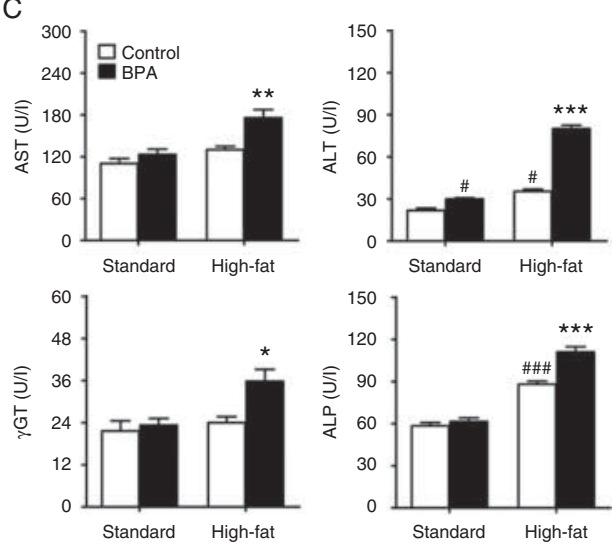

B
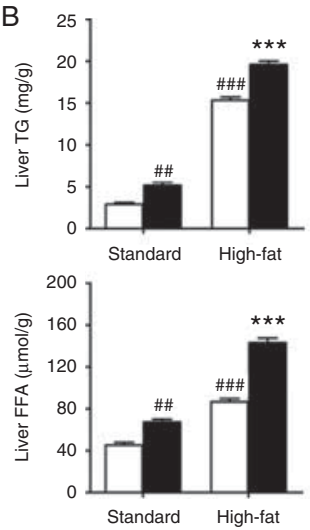

D
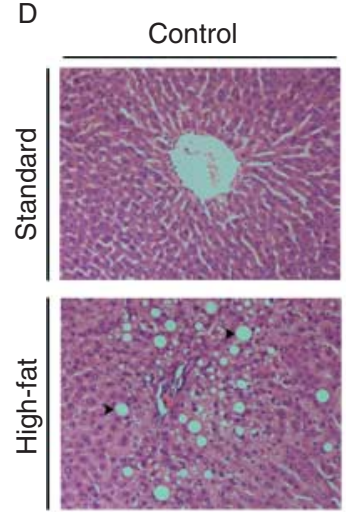
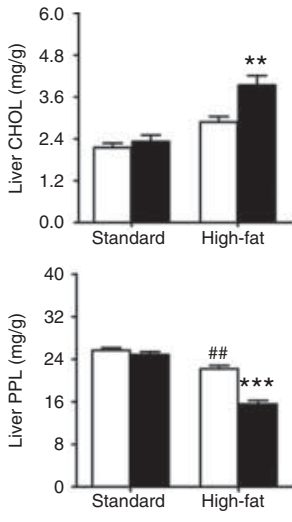

BPA
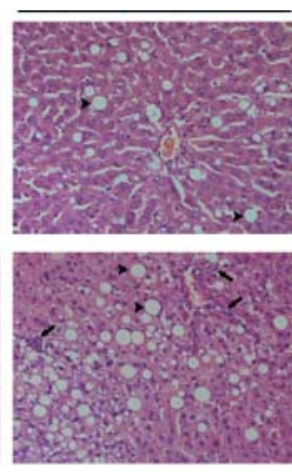

Figure 1

The effect of BPA on liver injury. (A) Body weight (wt), liver weight, and body liver percentage. (B) Hepatic lipid contents. Hepatic levels of triglycerides (TGs), cholesterol (CHOL), free fatty acids (FFAs), and phospholipids (PPLs) were measured. (C) Liver function tests. Serum level of aspartate aminotransferase (AST), alanine aminotransferase (ALT), $\gamma$-glutamyltransferase $(\gamma \mathrm{GT})$, and alkaline phosphatase (ALP) were measured. (D) Representative light microscopic micrographs of liver section

weight ratio respectively (Fig. 1A). For hepatic lipid content, TGs and FFAs were increased in offspring of rats exposed to BPA compared with controls, regardless of whether they were fed on a standard chow or HFD (Fig. 1B). Higher CHOL and lower PPLs were observed only in offspring of rats exposed to BPA fed on a HFD, compared with controls fed on a HFD (Fig. 1B).

\section{Hepatic function test}

On a SD, serum level of ALT was increased in offspring of rats exposed to BPA compared with controls (Fig. 1C), but no difference in AST, $\gamma \mathrm{GT}$, and ALP was observed between the offspring of control rats and the offspring of rats exposed to BPA. On a HFD, serum levels of ALT, AST, $\gamma \mathrm{GT}$, and ALP were higher in the offspring of rats exposed to BPA than in controls fed on a HFD (Fig. 1C), indicative of liver injury.

stained with hematoxylin and eosin dye (400 $\times$ magnification). Arrowheads indicate lipid droplets and arrows indicate inflammatory clusters. Data are expressed as means \pm s.E.M. ( $n=6$ rats per group); only one offspring was selected per litter. ${ }^{\#} P<0.05 ;{ }^{\# \#} P<0.01 ;{ }^{\# \# \#} P<0.001$ relative to the value for the control offspring fed on a SD. ${ }^{*} P<0.05$; ${ }^{* * P} P<0.01$; $* * * P<0.001$ relative to the value for the control offspring fed on a HFD. SD, standard chow diet; HFD, high-fat diet.

\section{Histological examination}

H\&E-stained liver sections from offspring of control rats fed on a SD revealed normal liver histology manifesting as normal cell size, with a prominent cell nucleus, uniform cytoplasm, and radially aligned hepatic cell cords, whereas the offspring of rats exposed to BPA fed on the same diet showed some features similar to those observed in human NAFLD, including disordered hepatic cell cords and modestly increased lipid droplets (Fig. 1D). On a HFD, an evident injury was observed in the livers of the offspring of both control rats and rats exposed to BPA including almost complete absence of sinus hepaticus, disordered hepatic cell cord, diffuse lipid droplets, and ballooning degeneration (Fig. 1D). But notably, fat was observed mainly as macrovesicular droplets in the offspring of rats exposed to BPA fed on a HFD. Meanwhile, a higher extent of

Published by Bioscientifica Ltd 
ballooning and inflammatory clusters was also displayed in the livers of the offspring of rats exposed to BPA compared with controls fed on a HFD (Fig. 1D).

\section{Levels of genes related to energy metabolism in liver}

Genes coding for key factors involved in energy metabolism were examined in the offspring of rats exposed to BPA and control rats. As shown in Fig. 2, both mRNA and protein expressions of lipogenic transcription factor SREBP1C (Srebf1) were significantly increased in the livers of the offspring of rats exposed to BPA, regardless of whether rats are fed on a SD or a HFD. Similarly, mRNA expression of its target gene Fasn was coordinately upregulated in the offspring of rats exposed to BPA.
The mRNA expressions of Pparg and Cd36 were elevated in BPA-exposed offspring compared with the control under high-fat dietary conditions (Fig. 2A). Although the levels of PPARA (Ppara) and one of its targets Cpt1a were compensatorily increased in the offspring of rats exposed to BPA fed on a SD, both genes were decreased in the offspring of rats exposed to BPA fed on a HFD compared with controls fed on a HFD (Fig. 2). Finally, two key regulators of hepatic gluconeogenesis, PCK1 and G6PC, were examined. It can be observed from Fig. 2 that BPA had no significant effect on expression of either PCK1 (Pck1) or G6PC (G6pc) in offspring fed on a SD. However, the mRNA and protein levels of both genes were increased in the offspring of rats exposed to BPA fed on a HFD compared with controls fed on a HFD.
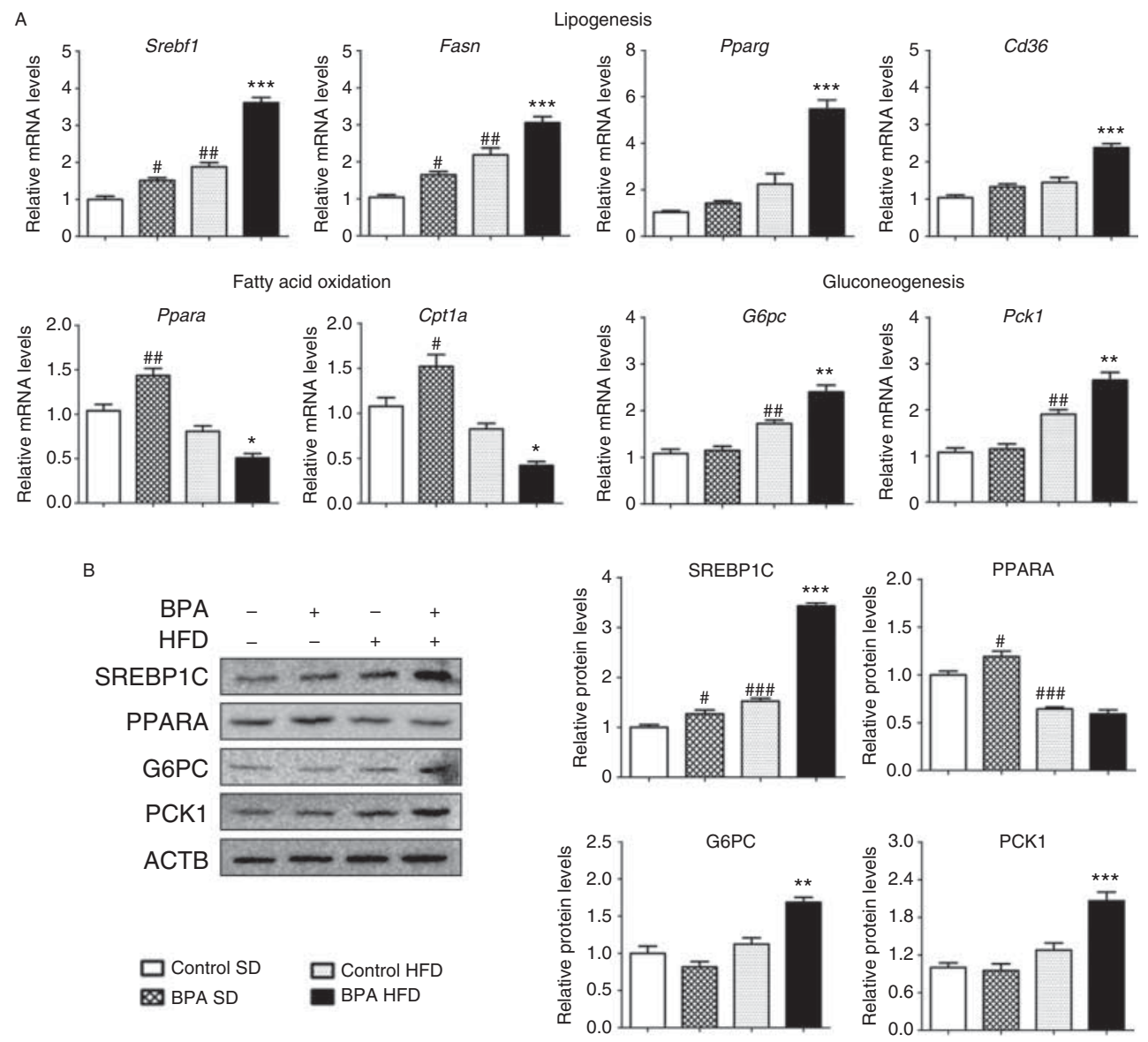

Figure 2

The effect of BPA on hepatic energy metabolism in the liver. (A) mRNA expression of genes associated with lipogenesis, fatty acid oxidation, and gluconeogenesis. (B) Levels of key proteins involved in hepatic energy metabolism. The expression levels were normalized to the ACTB internal control and were expressed as fold of values in control offspring fed on a SD. Data are expressed as means \pm s.E.M. For $m R N A, n=5$ rats per group,

three replicates for each gene; for protein, $n=3$ rats per group, three replicates for each gene; only one offspring was selected per litter. ${ }^{\#} P<0.05 ;{ }^{\# \#} P<0.01 ;{ }^{\# \#} P<0.001$ relative to the control offspring fed on a SD. ${ }^{*} P<0.05 ; * * P<0.01 ; * * * P<0.001$ relative to the control offspring fed on a HFD. SD, standard chow diet; HFD, high-fat diet. 


\section{Insulin signaling elements in the liver}

Hepatic steatosis is strongly associated with insulin resistance (Marchesini et al. 2005, Utzschneider \& Kahn 2006), hence the HOMA-IR and the QUICKI were calculated. It is shown in Fig. 3A that the HOMA-IR was higher, whereas the QUICKI was lower in the offspring of rats exposed to BPA compared with the offspring of control rats regardless of whether they were fed on a SD or a HFD. Perinatal exposure to BPA decreased hepatic insulin sensitivity and induced insulin resistance in the rat offspring. As insulin resistance could result from defective insulin signaling in peripheral tissues, the activation of insulin signal elements in the liver was determined by western blot in the rat offspring after insulin injection. It is shown in Fig. 3B that the levels of insulin-stimulated p-INSR, p-AKT1, and p-GSK3B were significantly downregulated in the livers of the offspring of rats exposed to BPA compared with controls, but the levels of INSR, AKT1, and GSK3B were similar between the offspring of control rats and the offspring of rats exposed to BPA, regardless of whether they were fed on a SD or a HFD. Meanwhile, p-INSR to INSR ratio, p-AKT1 to AKT1 ratio, and p-GSK3B to GSK3B ratio were also significantly less in BPA-exposed offspring than in the control offspring fed on the same diet (Fig. 3B).

\section{Hepatic oxidative stress}

Indexes related to oxidative stress were measured in the livers of rat offspring. On a SD, the offspring of rats exposed to BPA exhibited a lower GSH:GSSG ratio compared with controls (Fig. 4A). On a HFD, perinatal exposure to BPA increased liver GSSG levels in the offspring, with significant diminution in hepatic GSH and decreases in the GSH:GSSG ratio (Fig. 4A). The levels of a lipid peroxidation product MDA, another biomarker of oxidative stress, were significantly increased in the liver
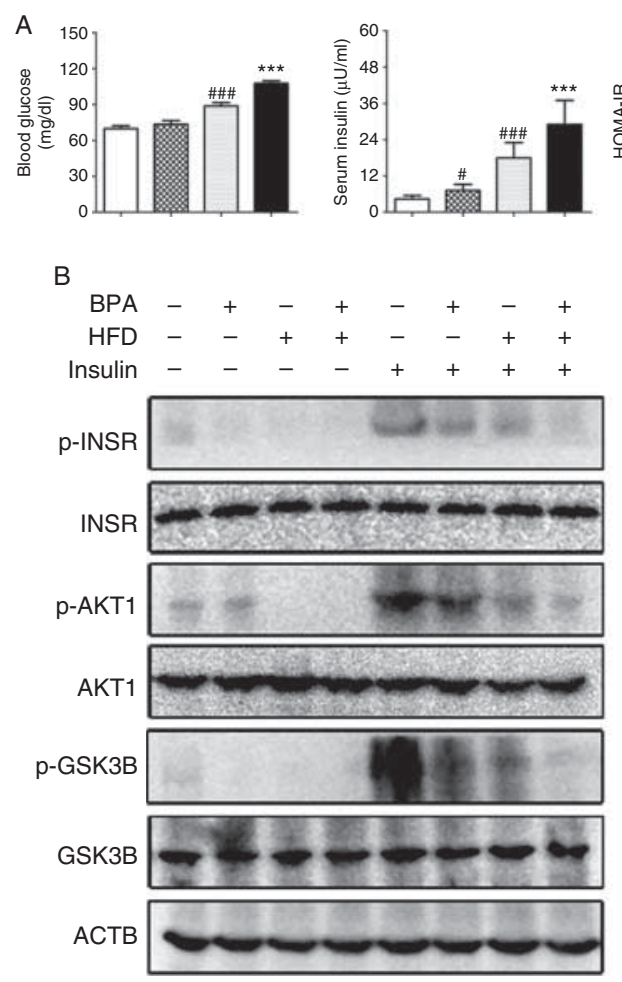
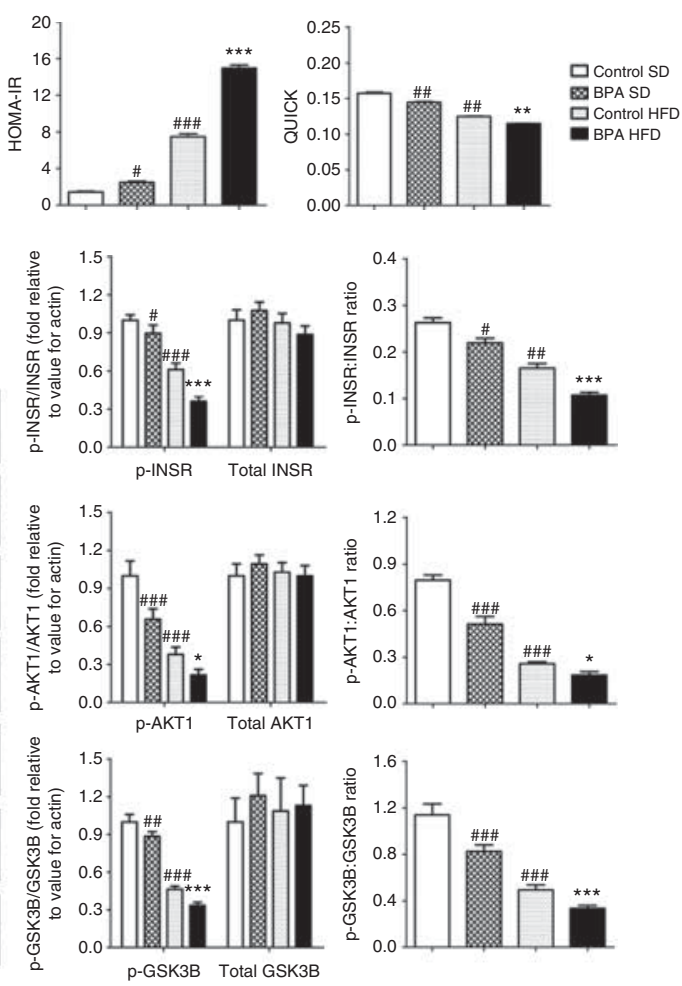

\section{Figure 3}

The effect of BPA on hepatic insulin resistance. (A) Fasting blood glucose, serum insulin, and the calculation of homeostasis model assessment of insulin resistance (HOMA-IR) and quantitative insulin sensitivity check index (QUICKI) ( $n=6$ rats per group; only one offspring was selected per litter). (B) Regulation of the key insulin signaling element protein phosphorylation in the liver. Rat offspring were fasted overnight and received intraperitoneal injections of saline or insulin $(0.75 \mathrm{U} / \mathrm{kg})$. Liver tissues were harvested $10 \mathrm{~min}$ later for immunoblotting analysis. http://joe.endocrinology-journals.org DOI: 10.1530/JOE-14-0356
(C) 2014 Society for Endocrinology Printed in Great Britain
Protein levels were quantified relative to a ACTB loading control and were expressed as fold of values in control offspring fed on a SD. Data are expressed as means \pm S.E.M. of three replicates for each protein $(n=3$ rats per group; only one offspring was selected per litter). ${ }^{\#} P<0.05,{ }^{\# \#} P<0.01$,

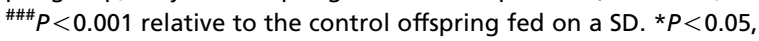
$* * P<0.01, * * * P<0.001$ relative to the control offspring fed on a HFD. SD, standard chow diet; HFD, high-fat diet.

Published by Bioscientifica Ltd. 


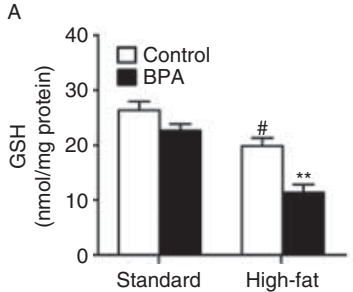

C
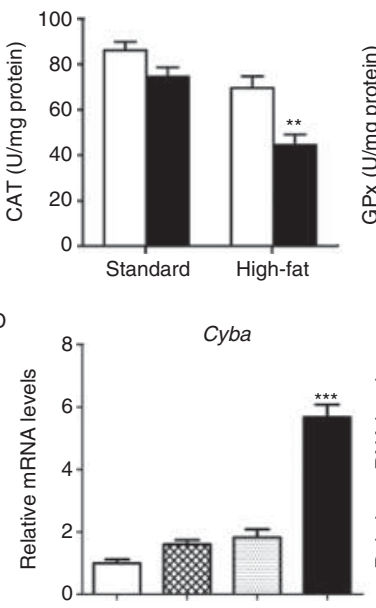
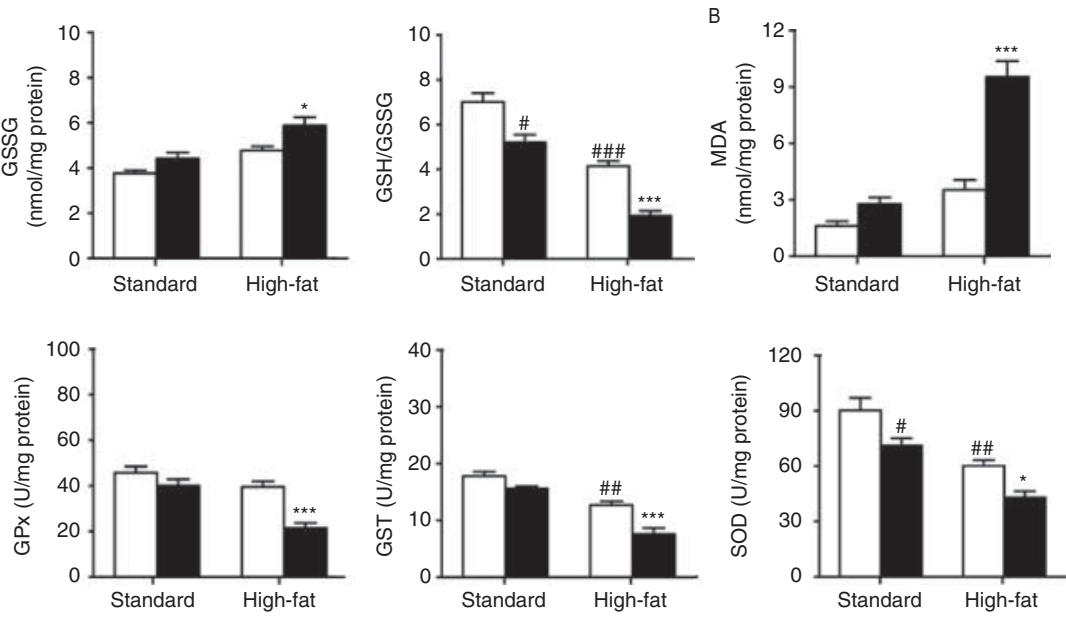

$y b b$
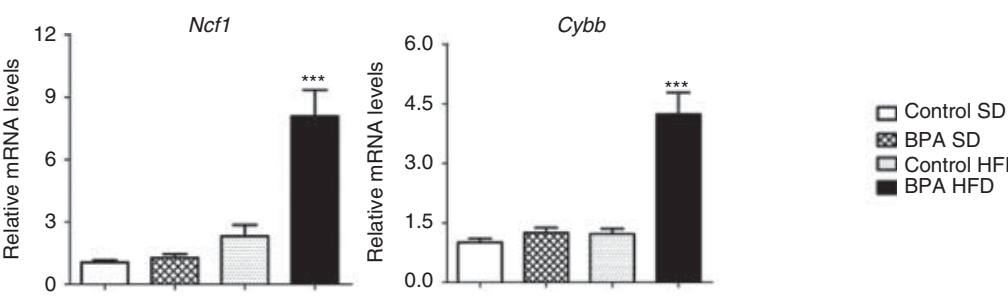

Control HFD

Control HFD

Figure 4

The effect of BPA on hepatic oxidative stress. (A) Glutathione (GSH) homeostasis. (B) Levels of malondialdehyde (MDA). (C) Activities of antioxidant enzymes, including catalase (CAT), glutathione peroxidase (GPx), glutathione S-transferase (GST), and superoxide dismutase (SOD). (D) NADPH oxidase subunit mRNA expression. Expression levels were normalized to the Actb internal control and were expressed as fold of

homogenates of the offspring of rats exposed to BPA compared with that measured in controls fed on a HFD (Fig. 4B). Meanwhile, the levels of the antioxidant enzymes, CAT, GPx, GST, and SOD, were reduced in the offspring of rats exposed to BPA fed on a HFD compared with controls fed on a HFD, as shown in Fig. 4C. Additionally, the mRNA expression of ROS-generating NADPH oxidases, such as $C y b a, N c f 1$, and $C y b b$, were significantly upregulated in the offspring of rats exposed to BPA fed on a HFD (Fig. 4D). No significant difference was detected in the mRNA expression of these genes between the offspring of control rats and the offspring of rats exposed to BPA fed on a SD (Fig. 4D).

\section{Inflammation and fibrosis}

On a SD, no difference in the serum inflammatory cytokines IL6 and TNF was observed between the offspring of rats exposed to BPA and the offspring of control rats (Fig. 5A). But on a HFD, elevated levels of both IL6 and TNF were shown in BPA-exposed offspring compared with values in control offspring fed on a SD. Data are expressed as means \pm s.E.M. (A, $B$, and $C$ ), $n=6$ rats per group; (D), $n=5$ rats per group, three replicates for each gene; only one offspring was selected per litter. ${ }^{\#} P<0.05$, ${ }^{\# \#} P<0.01,{ }^{\# \# \#} P<0.001$ relative to the control offspring fed on a SD. $* P<0.05, * * P<0.01, * * * P<0.001$ relative to the control offspring fed on a HFD. SD, standard chow diet; HFD, high-fat diet.

controls fed on a HFD (Fig. 5A). Consistent with the increased peripheral blood levels of inflammatory mediators, exposure to BPA also significantly increased the mRNA and protein levels of TNF and IL6 in the liver of offspring fed on a HFD (Fig. 5B). Using Masson's trichrome staining, results shown in Fig. 5C indicated that no significant histological signs of hepatic fibrosis appeared in offspring of both groups fed on the SD and the HFD. However, incipient perivenular fibrosis was observed in the livers of the offspring of rats exposed to BPA fed on a HFD (Fig. 5C). Consistent with staining results, both mRNA and protein levels of key fibrotic markers, including ACTA2, COL1A1, and TGFB1, were increased in the livers of the offspring of rats exposed to BPA fed on a HFD compared with controls fed on a HFD (Fig. 5D).

\section{Discussion}

BPA is one of the most widely used industrial chemicals with applications in the manufacture of plastic products that can increase the risk of chronic metabolic disease.

Published by Bioscientifica Ltd. 
A
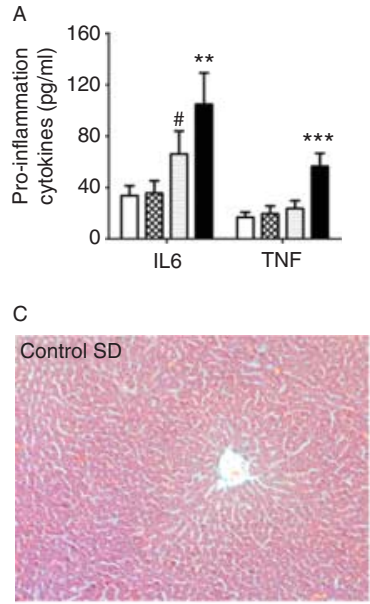

D

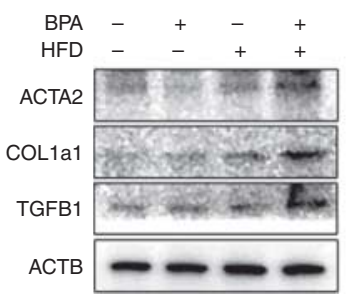

B

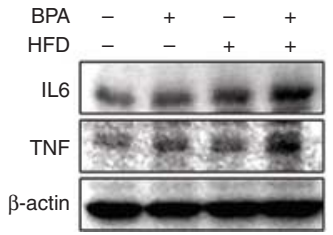

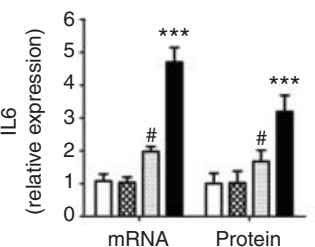

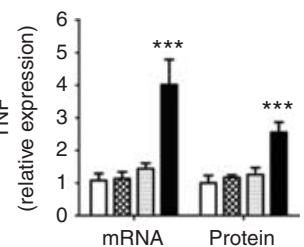

\section{Figure 5}

The effect of BPA on hepatic inflammation and fibrosis response. (A) Serum concentrations of inflammatory cytokines: IL6 and TNF $(n=6)$. (B) mRNA and protein levels of IL6 and TNF. (C) Representative Masson's trichrome stains of livers $(200 \times$ magnification). The collagen fibers are stained blue. (D) mRNA and protein levels of key fibrotic markers: ACTA2, COL1A1, and TGFB1. The expression levels were normalized to the ACTB internal control and were expressed as fold of values in control offspring fed

With regard to this finding, we had previously shown that perinatal exposure to BPA resulted in multiple features of metabolic syndrome in the offspring, including increased body weight, hyperlipidemia, hyperleptinemia, and insulin resistance (Wei et al. 2011). Considering that NAFLD is the typical hepatic manifestation of the metabolic syndrome, the current study further investigated the adverse effects of BPA on the liver.

In this study, perinatal exposure to BPA, even at the current TDI of $50 \mu \mathrm{g} / \mathrm{kg}$ per day, induced abnormal accumulation of lipids in the liver of adult offspring. These findings were in agreement with results described in a previous paper demonstrating that male CD1 mice treated with BPA at $50 \mu \mathrm{g} / \mathrm{kg}$ per day for 28 days exhibited accumulation of CHOLs and TGs in the liver (Marmugi et al. 2012). Hepatic steatosis results from a disturbance in the balance among TG delivery, synthesis, export, and oxidation. In the liver, TGs are assembled by coupling fatty acids (FAs) to a glycerol backbone via ester bonds (Cohen
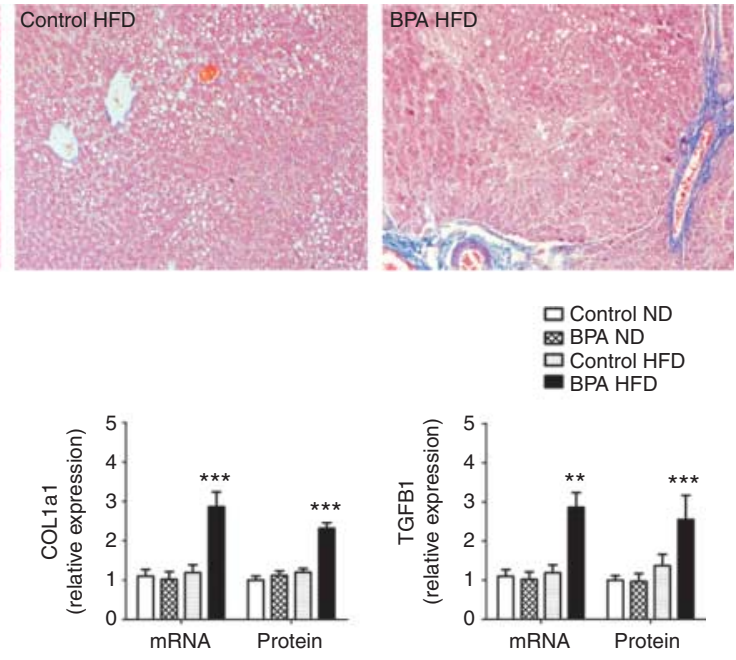

on a SD. Data are expressed as means \pm S.E.M. For $\mathrm{mRNA}, n=5$ rats per group, three replicates for each gene; for protein, $n=3$ rats per group, three replicates for each gene; only one offspring was selected per litter. ${ }^{\#} P<0.05$ relative to the control offspring fed on a SD. ${ }^{*} P<0.05, * * P<0.01$, $* * * P<0.001$ relative to the control offspring fed on a HFD. SD, standard chow diet; HFD, high-fat diet.

et al. 2011). Alterations in FA source, including circulating FFAs from adipose tissue via lipolysis (59\%), newly produced FAs within the liver through de novo lipogenesis (26\%), and dietary FAs (15\%) (Donnelly et al. 2005), would produce a fatty liver phenotype. In a pervious study, we had observed that BPA exposure resulted in a higher body fat percentage and a significantly greater mass of adipocytes in both offspring fed on a SD and those fed on a HFD. The expansion of adipose tissue mediated by exposure to BPA made the organism release excess FFAs into the circulation (Supplementary Figure 1, see section on supplementary data given at the end of this article) and, moreover, diminished the release of insulin-sensitizing and antiinflammatory cytokines and increased expression of proinflammatory molecules, finally resulting in fatty liver and insulin resistance. Also, SREBP1C and its downstream lipogenic targets (FASN, PPARG, and CD36) were stimulated in the livers of the offspring of rats exposed to BPA, indicating that BPA increased lipogenesis via prompting

Published by Bioscientifica Ltd 
de novo synthesis of FAs. Specifically, a significantly higher degree of lipid accumulation, together with impaired liver function, widespread ballooning degeneration, moderate inflammatory cell infiltration, and mild fibrosis, was exhibited in the livers of the offspring of rats exposed to BPA when they consumed a HFD immediately after weaning. A study conducted on rats demonstrated that dietary fats taken up in the intestine could be packaged into TG-rich chylomicrons and 20\% of TGs in chylomicrons were then delivered to the liver (Redgrave 1970). Extrapolating from these experiments, the HFD $(20 \mathrm{~g}$ food per day) could furnish the liver with a significantly higher amount of fat (approximately $0.53 \mathrm{~g}$ fat each day) than the SD (approximately $0.18 \mathrm{~g}$ fat each day). Postweaning HFD intake was responsible, at least in part, for excess FA supply to the liver.

In response to the large pool of FAs, genes involved in the FA $\beta$-oxidation pathway, such as Ppara and its target gene Cpt1a, were altered by BPA in livers of offspring. Ppara is the predominant PPAR subtype in rodent liver, which plays a major role in controlling genes involved in FA elongation, desaturation, oxidation, and transport (Reddy 2001). Cpt1a is a membrane transporter of FAs from the cytoplasm into the mitochondrial matrix and is a primary rate-limiting enzyme involved in FA oxidation (McGarry \& Foster 1980). Although exposure to BPA caused unexpected activation of Ppara and Cpt1a in offspring fed on a SD, it may be only a compensatory response to metabolize the excessive FAs in the liver. In contrast to these findings, the offspring of rats exposed to BPA fed on a HFD had a notable reduction in both Ppara and Cpt1a, which was indicative of inhibition of FA oxidation and which in turn would further lead to hepatic lipid accumulation. BPA adversely influenced the coordinated modulation of hepatic uptake, synthesis, and oxidation of FAs, thereby causing accumulation of toxic lipid-derived metabolites in hepatocytes.

In this study, marked increases in HOMA-IR and decreases in QUICKI were established in the offspring of rats exposed to BPA, indicating that perinatal exposure to BPA induced insulin resistance in the rat offspring. The liver is a key organ for glucose and lipid metabolism, hence we subsequently measured whether exposure to BPA induced insulin resistance by blocking the hepatocellular insulin signaling pathway. We found that autophosphorylation of the INSR on Tyr1150/1151 in response to insulin was decreased in the livers of offspring after BPA treatment compared with controls, which was followed by reduced AKT1 phosphorylation on Thr308 and its downstream GSK3B phosphorylation on Ser9.
These observations were in agreement with results reported in a previous paper in which the level of tyrosine phosphorylation of the INSR was decreased in the livers of mice exposed to $100 \mu \mathrm{g} / \mathrm{kg}$ per day BPA for 8 days (Batista et al. 2012). However, insulin-induced AKT1 phosphorylation was similar for BPA-exposed and control mice in that study, which may be due to a compensation mechanism that occurred at the early stage of insulin resistance, before the onset of hyperglycemia. In this study, BPA-exposed offspring had developed a higher degree of insulin resistance, characterized by hyperglycemia, hyperinsulinemia, and glucose intolerance. We thus suggested that BPA decreased expression of p-AKT1 and p-GSK3B in the livers of offspring, thereby leading to an increase in the hepatic glucose output and finally contributing to the continuous hyperglycemia. In addition, two gluconeogenic key enzymes, G6PC and PCK1, were elevated in the livers of the offspring of rats exposed to BPA fed on a HFD. Normally, insulin is able to rapidly and substantially inhibit G6PC and PCK1 and gluconeogenesis in the liver. However, the offspring of rats exposed to BPA fed on a HFD exhibited lack of suppression of these gene transcription processes during hyperinsulinemia. Glucose overproduction would lead to further increases in insulin secretion, finally contributing to the vicious cycle that triggered the liver to become highly insulin-resistant.

Notably, AKT1 is known to be a major regulator of lipogenesis in the liver. AKT1 activates SREBP1C via AKT1mediated phosphorylation and inhibition of GSK3. In this study, exposure to BPA significantly impaired AKT1 signaling in the liver. Under these circumstances, it appeared that transcriptional activity of SREBP1C might be repressed by increased GSK3 activity, leading to the reduced expression of downstream lipogenic genes. But surprisingly, hepatic TGs and lipogenic gene expression were increased after exposure to BPA even in the absence of hepatic insulin signaling, indicating that insulin resistance (impaired activation of AKT1 pathway) and hypersensitivity to insulin (increased expression of SREBP1C) existed concomitantly in the livers of the offspring of rats exposed to BPA. These results were consistent with the observation that the expression of SREBP1C was increased, whereas the insulin-induced activation of AKT1 was inhibited in the livers of $o b / o b$ mice (Shimomura et al. 2000). We interpreted these data to indicate that hyperinsulinemia was able to stimulate SREBP1C activity in the livers of the offspring of animals exposed to BPA through a pathway that did not require AKT1 activation. Further investigations are still required to explore the signaling molecule involved in promoting SREBP1C activation.

Published by Bioscientifica Ltd 
According to the developmental origin hypothesis, we suggested that exposure to BPA during critical stages of development made the offspring develop responses to altered intrauterine environments, ultimately predisposing them to fatty liver disease. Furthermore, developmental programing mediated by BPA affected postnatal defensive responses to other stresses (such as HFD) in offspring. When the offspring of rats exposed to BPA were weaned onto a HFD, several histopathological features reminiscent of human NASH, such as micro- and macro-vesicular lipid accumulation, ballooning, inflammation infiltration, the gradual appearence of collagen fibrils, and impaired cell function, were detected in the liver. Aberrant epigenetic modifications have been suggested to be one of the important biological mechanisms linking fetal exposures to metabolic disease progression. The epigenome can control and influence gene expression profiles of most organs and cell types during development, both in utero and throughout life, through DNA methylation, histone modifications, and a variety of non-coding RNAs (Bernal \& Jirtle 2010). BPA has been reported to be epigenetically toxic, which can induce aberrant epigenetic modifications in early life (Singh \& Li 2012, Veiga-Lopez et al. 2013). For example, perinatal exposure to $50 \mu \mathrm{g} / \mathrm{kg}$ per day BPA decreased hepatic global DNA methylation and increased expression of DNA methyltransferase 3B mRNA in male rat offspring at 3 and 21 weeks. Alterations in DNA methylation regulated the expression of certain important genes such as $G c k$, consequently contributing to insulin resistance and impaired glucose intolerance in adulthood (Ma et al. 2013). In addition, exposure to BPA has also been reported to interfere with DNA repair in the placental cell line through alterations of cellular microRNA, further supporting the potential role of epigenetics in fetal reprograming by BPA-induced toxicity (Avissar-Whiting et al. 2010). Thus, we postulated that the BPA-induced fatty liver phenotype may also be epigenetically associated, although the way in which BPA affects the epigenetic process is unclear and remains to be demonstrated in future studies.

In this study, another mechanism of the liver pathology of steatohepatitis observed in BPA-exposed offspring upon a feeding with a high-fat diet was oxidative stress and its downstream events, as we identified accumulation of MDA. Perinatal exposure to BPA induced hepatic oxidative stress in offspring fed on a HFD, possibly via the upregulation of genes involved in the generation of ROS (e.g. the NADPH oxidase family $C y b a, N c f 2$, and $C y b b$ ) and the reduction in both enzymatic and nonenzymatic antioxidants. ROS along with the products of

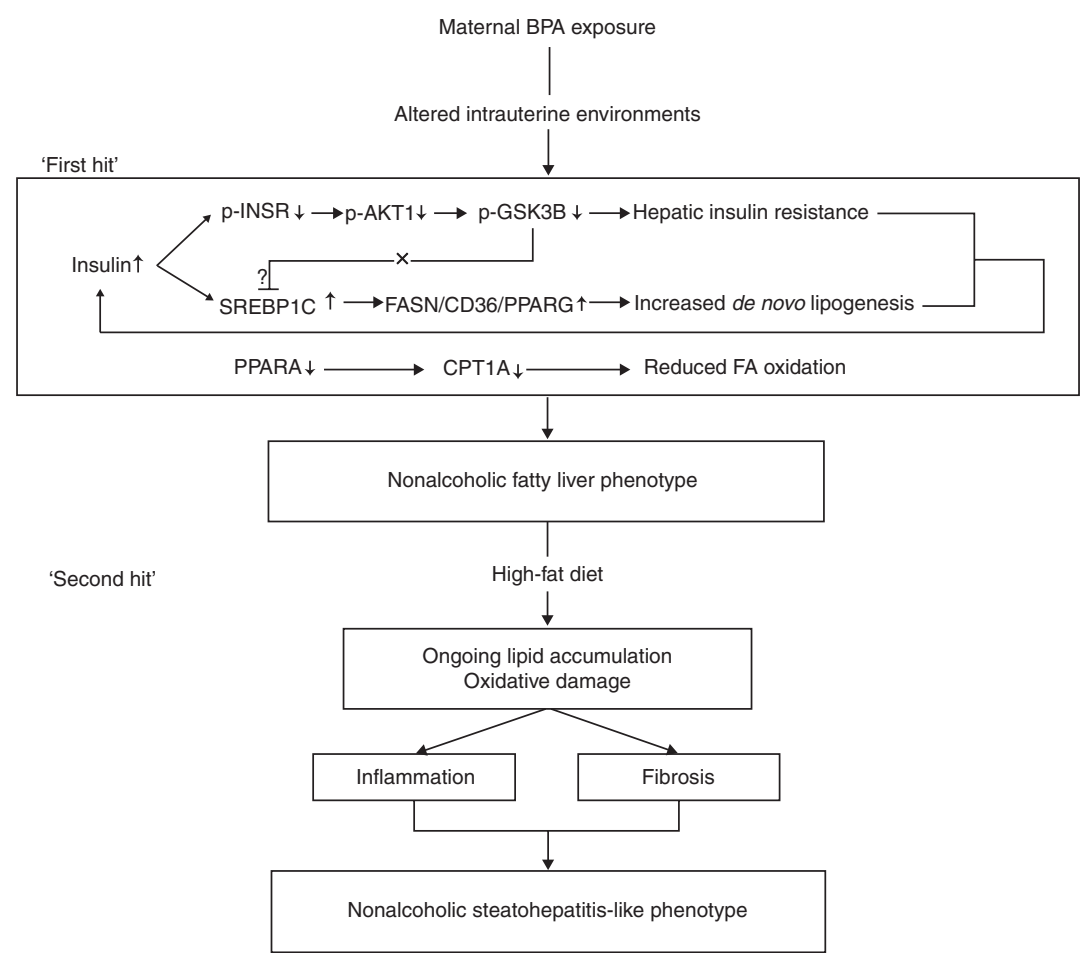

Figure 6

Proposed pathways by which fatty liver disease is programed in BPA-exposed offspring.

http://joe.endocrinology-journals.org DOI: 10.1530/JOE-14-0356
C 2014 Society for Endocrinology Printed in Great Britain
Published by Bioscientifica Ltd. 
lipid peroxidation could activate nuclear factor $\kappa \mathrm{B}$, which in turn induced the release of several cytokines, including TNF, IL6, TGFB1, etc., in the BPA-exposed offspring upon high-fat feeding. Upregulation of proinflammatory cytokines TNF and IL6 could induce hepatic inflammation through delivering polymorphonuclear and mononuclear leukocytes into inflamed tissues, and increased TGFB1 was able to activate hepatic stellate cells into collagenproducing myofibroblastic cells, promoting collagen synthesis in the liver. TNF and lipid peroxidation products could also inhibit the electron transport chain of the mitochondria and worsen the mitochondrial function (Nagakawa et al. 2005). Mitochondrial damage in turn would lead to secondary production of ROS and inhibition of the $\beta$-oxidation of lipids (Santra et al. 2007), further increasing the progression of hepatic steatosis, inflammation, and fibrosis and starting a vicious circle.

In summary, origins of metabolic diseases may lie in early life, even in utero, experiences. Perinatal exposure to BPA led to an excess of lipid accumulation and insulin resistance in the livers of the rat offspring. More importantly, perinatal exposure to BPA also resulted in the liver being 'primed' for more severe steatosis, inflammation, and fibrosis. The postweaning HFD triggered a steatohepatitis phenotype in the offspring of rats exposed to BPA via aggravation of hepatic oxidative stress (Fig. 6).

\section{Supplementary data}

This is linked to the online version of the paper at http://dx.doi.org/10.1530/ JOE-14-0356.

\section{Declaration of interest}

The authors declare that there is no conflict of interest that could be perceived as prejudicing the impartiality of the research reported.

\section{Funding}

This work was supported by the National Program on Key Basic Research Project of China (973 Program) (2012CB722401), the National Natural Science Foundation of China (81030051, 21177046, and 21207128), the R\&D Special Fund for Public Welfare Industry (Environment) (201309048), the National Basic Research Development Program of China (2008CB418206), the Fundamental Research Funds for the Central Universities, Huazhong University of Science and Technology (2012QN240 and 2012TS072), and the Natural Science Foundation of Fujian Province, China (2013J05033).

\section{Author contribution statement}

$\mathrm{S} X$ conceived and designed the study. J $\mathrm{W}$ and $\mathrm{Y} L$ performed the researches and wrote the manuscript. X $S$ carried out all western blot experiments and participated in data processing and statistical analyses. $Y L$ reviewed and edited the manuscript. $L S, Z Z$, and $B X$ participated in animal experiments and data processing. $\mathrm{Y} C$ carried out additional experiments in the revision of the manuscript. All authors have contributed to, seen, and approved the manuscript.

\section{Acknowledgements}

The authors thank the Animal Laboratory of Wuhan University for providing animal management and care.

\section{References}

Alonso-Magdalena P, Vieira E, Soriano S, Menes L, Burks D, Quesada I \& Nadal A 2010 Bisphenol A exposure during pregnancy disrupts glucose homeostasis in mothers and adult male offspring. Environmental Health Perspectives 118 1243-1250. (doi:10.1289/ehp.1001993)

Alonso-Magdalena P, Quesada I \& Nadal A 2011 Endocrine disruptors in the etiology of type 2 diabetes mellitus. Nature Reviews. Endocrinology 7 346-353. (doi:10.1038/nrendo.2011.56)

Avissar-Whiting M, Veiga KR, Uhl KM, Maccani MA, Gagne LA, Moen EL \& Marsit CJ 2010 Bisphenol A exposure leads to specific microRNA alterations in placental cells. Reproductive Toxicology 29 401-406. (doi:10.1016/j.reprotox.2010.04.004)

Azzalini L, Ferrer E, Ramalho LN, Moreno M, Domínguez M, Colmenero J, Peinado VI, Barberà JA, Arroyo V \& Ginès P 2010 Cigarette smoking exacerbates nonalcoholic fatty liver disease in obese rats. Hepatology $\mathbf{5 1}$ 1567-1576. (doi:10.1002/hep.23516)

Batista TM, Alonso-Magdalena P, Vieira E, Amaral MEC, Cederroth CR, Nef S, Quesada I, Carneiro EM \& Nadal A 2012 Short-term treatment with bisphenol-A leads to metabolic abnormalities in adult male mice. PLOS ONE 7 e33814. (doi:10.1371/journal.pone.0033814)

Bayol SA, Simbi BH, Fowkes RC \& Stickland NC 2010 A maternal “junk food" diet in pregnancy and lactation promotes nonalcoholic fatty liver disease in rat offspring. Endocrinology 151 1451-1461. (doi:10.1210/en.2009-1192)

Bernal AJ \& Jirtle RL 2010 Epigenomic disruption: the effects of early developmental exposures. Birth Defects Research. Part A, Clinical and Molecular Teratology 88 938-944. (doi:10.1002/bdra.20685)

Bonora E, Targher G, Alberiche M, Bonadonna RC, Saggiani F, Zenere MB, Monauni T \& Muggeo M 2000 Homeostasis model assessment closely mirrors the glucose clamp technique in the assessment of insulin sensitivity: studies in subjects with various degrees of glucose tolerance and insulin sensitivity. Diabetes Care 23 57-63. (doi:10.2337/diacare. 23.1.57)

Chan SM, Sun R-Q, Zeng X-Y, Choong Z-H, Wang H, Watt MJ \& Ye J-M 2013 Activation of $\operatorname{PPAR} \alpha$ ameliorates hepatic insulin resistance and steatosis in high fructose - fed mice despite increased endoplasmic reticulum stress. Diabetes 62 2095-2105. (doi:10.2337/db12-1397)

Chen H, Sullivan G \& Quon MJ 2005 Assessing the predictive accuracy of QUICKI as a surrogate index for insulin sensitivity using a calibration model. Diabetes 54 1914-1925. (doi:10.2337/diabetes.54.7.1914)

Cohen JC, Horton JD \& Hobbs HH 2011 Human fatty liver disease: old questions and new insights. Science 332 1519-1523. (doi:10.1126/ science.1204265)

Diamanti-Kandarakis E, Bourguignon J-P, Giudice LC, Hauser R, Prins GS, Soto AM, Zoeller RT \& Gore AC 2009 Endocrine-disrupting chemicals: an Endocrine Society scientific statement. Endocrine Reviews 30 293-342. (doi:10.1210/er.2009-0002)

Donnelly KL, Smith CI, Schwarzenberg SJ, Jessurun J, Boldt MD \& Parks EJ 2005 Sources of fatty acids stored in liver and secreted via lipoproteins in patients with nonalcoholic fatty liver disease. Journal of Clinical Investigation 115 1343-1351. (doi:10.1172/JCI23621)

Fan JG, Zhu J, Li XJ, Chen L, Lu YS, Li L, Dai F, Li F \& Chen SY 2005 Fatty liver and the metabolic syndrome among Shanghai adults. Journal of 
Gastroenterology and Hepatology 20 1825-1832. (doi:10.1111/j.14401746.2005.04058.x)

Farrell GC \& Larter CZ 2006 Nonalcoholic fatty liver disease: from steatosis to cirrhosis. Hepatology 43 S99-S112. (doi:10.1002/hep.20973)

Folch J, Lees M \& Sloane-Stanley G 1957 A simple method for the isolation and purification of total lipids from animal tissues. Journal of Biological Chemistry 226 497-509.

Friedman TC, Sinha-Hikim I, Parveen M, Najjar SM, Liu Y, Mangubat M, Shin C-S, Lyzlov A, Ivey R \& Shaheen M 2012 Additive effects of nicotine and high-fat diet on hepatic steatosis in male mice. Endocrinology 153 5809-5820. (doi:10.1210/en.2012-1750)

Hamaguchi M, Kojima T, Takeda N, Nakagawa T, Taniguchi H, Fujii K, Omatsu T, Nakajima T, Sarui H \& Shimazaki M 2005 The metabolic syndrome as a predictor of nonalcoholic fatty liver disease. Annals of Internal Medicine 143 722-728. (doi:10.7326/0003-4819-143-10200511150-00009)

Huc L, Lemarié A, Guéraud F \& Héliès-Toussaint C 2012 Low concentrations of bisphenol A induce lipid accumulation mediated by the production of reactive oxygen species in the mitochondria of HepG2 cells. Toxicology In Vitro 26 709-717. (doi:10.1016/j.tiv.2012.03.017)

Katz A, Nambi SS, Mather K, Baron AD, Follmann DA, Sullivan G \& Quon MJ 2000 Quantitative insulin sensitivity check index: a simple, accurate method for assessing insulin sensitivity in humans. Journal of Clinical Endocrinology and Metabolism 85 2402-2410. (doi:10.1210/ jcem.85.7.6661)

Lang IA, Galloway TS, Scarlett A, Henley WE, Depledge M, Wallace RB \& Melzer D 2008 Association of urinary bisphenol A concentration with medical disorders and laboratory abnormalities in adults. Journal of the American Medical Association 300 1303-1310. (doi:10.1001/jama.300. 11.1303)

Ma Y, Xia W, Wang D, Wan Y, Xu B, Chen X, Li Y \& Xu S 2013 Hepatic DNA methylation modifications in early development of rats resulting from perinatal BPA exposure contribute to insulin resistance in adulthood. Diabetologia 56 2059-2067. (doi:10.1007/s00125-013-2944-7)

Marchesini G, Marzocchi R, Agostini F \& Bugianesi E 2005 Nonalcoholic fatty liver disease and the metabolic syndrome. Current Opinion in Lipidology 16 421-427. (doi:10.1097/01.mol.0000174153.53683.f2)

Marmugi A, Ducheix S, Lasserre F, Polizzi A, Paris A, Priymenko N, Bertrand-Michel J, Pineau T, Guillou H \& Martin PG 2012 Low doses of bisphenol A induce gene expression related to lipid synthesis and trigger triglyceride accumulation in adult mouse liver. Hepatology $\mathbf{5 5}$ 395-407. (doi:10.1002/hep.24685)

Matthews D, Hosker J, Rudenski A, Naylor B, Treacher D \& Turner R 1985 Homeostasis model assessment: insulin resistance and $\beta$-cell function from fasting plasma glucose and insulin concentrations in man. Diabetologia 28 412-419. (doi:10.1007/BF00280883)

McCurdy CE, Bishop JM, Williams SM, Grayson BE, Smith MS, Friedman JE \& Grove KL 2009 Maternal high-fat diet triggers lipotoxicity in the fetal livers of nonhuman primates. Journal of Clinical Investigation 119 323-335. (doi:10.1172/JCI32661)

McGarry J \& Foster D 1980 Regulation of hepatic fatty acid oxidation and ketone body production. Annual Review of Biochemistry 49 395-420. (doi:10.1146/annurev.bi.49.070180.002143)

Nagakawa Y, Williams GM, Zheng Q, Tsuchida A, Aoki T, Montgomery RA, Klein AS \& Sun Z 2005 Oxidative mitochondrial DNA damage and deletion in hepatocytes of rejecting liver allografts in rats: role of TNF- $\alpha$. Hepatology 42 208-215. (doi:10.1002/hep.20755)

Oben JA, Mouralidarane A, Samuelsson A-M, Matthews PJ, Morgan ML, Mckee C, Soeda J, Fernandez-Twinn DS, Martin-Gronert MS \& Ozanne SE 2010 Maternal obesity during pregnancy and lactation programs the development of offspring non-alcoholic fatty liver disease in mice. Journal of Hepatology 52 913-920. (doi:10.1016/j.jhep. 2009.12.042)
Reddy JK III 2001 Peroxisomal $\beta$-oxidation, PPAR $\alpha$, and steatohepatitis. American Journal of Physiology. Gastrointestinal and Liver Physiology 281 G1333-G1339.

Redgrave T 1970 Formation of cholesteryl ester-rich particulate lipid during metabolism of chylomicrons. Journal of Clinical Investigation 49465. (doi:10.1172/JCI106255)

Rubin BS \& Soto AM 2009 Bisphenol A: perinatal exposure and body weight. Molecular and Cellular Endocrinology 304 55-62. (doi:10.1016/ j.mce.2009.02.023)

Santra A, Chowdhury A, Ghatak S, Biswas A \& Dhali GK 2007 Arsenic induces apoptosis in mouse liver is mitochondria dependent and is abrogated by $N$-acetylcysteine. Toxicology and Applied Pharmacology 220 146-155. (doi:10.1016/j.taap.2006.12.029)

Shankar A, Teppala S \& Sabanayagam C 2012 Bisphenol A and peripheral arterial disease: results from the NHANES. Environmental Health Perspectives 120 1297-1300. (doi:10.1289/ehp.1104114)

Shen L, Liu Z, Gong J, Zhang L, Wang L, Magdalou J, Chen L \& Wang H 2014 Prenatal ethanol exposure programs an increased susceptibility of non-alcoholic fatty liver disease in female adult offspring rats. Toxicology and Applied Pharmacology 274 263-273. (doi:10.1016/ j.taap.2013.11.009)

Shimomura I, Matsuda M, Hammer RE, Bashmakov Y, Brown MS \& Goldstein JL 2000 Decreased IRS-2 and increased SREBP-1c lead to mixed insulin resistance and sensitivity in livers of lipodystrophic and $o b / o b$ mice. Molecular Cell 6 77-86. (doi:10.1016/S10972765(00)00009-5)

Singh S \& Li SS 2012 Epigenetic effects of environmental chemicals bisphenol A and phthalates. International Journal of Molecular Sciences 13 10143-10153. (doi:10.3390/ijms130810143)

Somm E, Schwitzgebel VM, Toulotte A, Cederroth CR, Combescure C, Nef S, Aubert ML \& Hüppi PS 2009 Perinatal exposure to bisphenol A alters early adipogenesis in the rat. Environmental Health Perspectives 117 1549-1555. (doi:10.1289/ehp.11342)

Tan H-H, Fiel MI, Sun Q, Guo J, Gordon RE, Chen L-C, Friedman SL, Odin JA \& Allina J 2009 Kupffer cell activation by ambient air particulate matter exposure may exacerbate non-alcoholic fatty liver disease. Journal of Immunotoxicology 6 266-275. (doi:10.3109/ 15476910903241704)

Tomaru M, Takano H, Inoue K-I, Yanagisawa R, Osakabe N, Yasuda A, Shimada A, Kato Y \& Uematsu H 2007 Pulmonary exposure to diesel exhaust particles enhances fatty change of the liver in obese diabetic mice. International Journal of Molecular Medicine 19 17-22. (doi:10.3892/ ijmm.19.1.17)

Tong M, Neusner A, Longato L, Lawton M, Wands JR \& de la Monte SM 2009 Nitrosamine exposure causes insulin resistance diseases: relevance to type 2 diabetes mellitus, non-alcoholic steatohepatitis, and Alzheimer's disease. Journal of Alzheimer's Disease 17 827-844. (doi:10.3233/JAD-2009-1155)

Utzschneider KM \& Kahn SE 2006 Review: The role of insulin resistance in nonalcoholic fatty liver disease. Journal of Clinical Endocrinology and Metabolism 91 4753-4761. (doi:10.1210/jc.2006-0587)

Veiga-Lopez A, Luense LJ, Christenson LK \& Padmanabhan V 2013 Developmental programming: gestational bisphenol-A treatment alters trajectory of fetal ovarian gene expression. Endocrinology 154 1873-1884. (doi:10.1210/en.2012-2129)

Vom Saal FS, Nagel SC, Coe BL, Angle BM \& Taylor JA 2012 The estrogenic endocrine disrupting chemical bisphenol A (BPA) and obesity. Molecular and Cellular Endocrinology 354 74-84. (doi:10.1016/j.mce. 2012.01.001)

Wei J, Lin Y, Li Y, Ying C, Chen J, Song L, Zhou Z, Lv Z, Xia W \& Chen X 2011 Perinatal exposure to bisphenol A at reference dose predisposes offspring to metabolic syndrome in adult rats on a high-fat diet. Endocrinology 152 3049-3061. (doi:10.1210/en.2011-0045)

Received in final form 6 June 2014

Accepted 1 July 2014 http://joe.endocrinology-journals.org DOI: $10.1530 / J O E-14-0356$
() 2014 Society for Endocrinology Printed in Great Britain 